\title{
Temporal coherence and correlation of counterpropagating twin photons
}

\author{
Alessandra Gatti ${ }^{1,2}$, Tommaso Corti $^{2}$, Enrico Brambilla ${ }^{2}$, \\ 1 Istituto di Fotonica e Nanotecnologie del CNR, Piazza Leonardo Da Vinci 32, Milano, \\ Italy; ${ }^{2}$ Dipartimento di Scienza e Alta Tecnologia dell' Università dell'Insubria, Via Valleggio 11, Como, Italy
}

\begin{abstract}
This work analyses the temporal coherence and correlation of counterpropagating twin photons generated in a quasi-phase matched nonlinear cristal by spontaneous parametric-down conversion. We find out different pictures depending on the pump pulse duration relative to two characteristic temporal scales, determined respectively by the temporal separation between the counterpropagating and the co-propagating wavepackets. When the pump duration is intermediate between the two scales, we show a transition from a highly entangled state to an almost separable state, with strongly asymmetric spectral properties of the photons.
\end{abstract}

\section{Introduction}

Spontaneous parametric down-conversion (SPDC) occurring in $\chi^{(2)}$ media is one of the most accessible sources both of entangled photon pairs and of single photons, heralded by detection of the partner. The microscopic process, where a high energy photon of the pump laser splits into two lower energy photons, is ruled by conservation laws (energy, momentum, angular momentum, polarization), which are at the origin of a wide range of quantum correlations between the members of the pair.

In standard co-propagating configurations, the two-photon state is characterized by a high dimensional entanglement, because a quantum correlation is present over huge temporal and angular bandwidths. The temporal correlation was historically the first one to be studied [1: a down-conversion event can take place anywhere along the crystal, so that the arrival time of the twins is not known. However, the members of a pair, generated at the same point, propagate nearly in the same direction, and exit the crystal almost simoultaneously A small uncertainty in their temporal separation is present because of their different group velocities (type II) or because of the group velocity dipersion (typeI), and can be reduced to its smallest limit (the optical cycle) when the spatial degrees of freedom are properly controlled [2, [3]. Such a short correlation time results in a high-dimensional temporal entanglement [5]. Its spectral counterpart is the huge spectral bandwidth of SPCD emission, and the high dimensional spectral entanglement of SPDC photons [6]. High-dimensional entanglement offers relevant opportunities in view of broadband quantum communication schemes, but can also be regarded as a negative feature, because it affects the purity of heralded single photons.

This work considers a non-conventional configuration, where one of the down-converted photons is generated in the backward direction with respect to the pump laser, in a periodically poled crystal (Fig 11). Although predicted almost fifty years ago [7, counter-propagating down-conversion has been only recently realized [8, 9], thanks to technical advancements in achieving the sub-micrometer poling periods necessary to phase-match the interaction [10] .

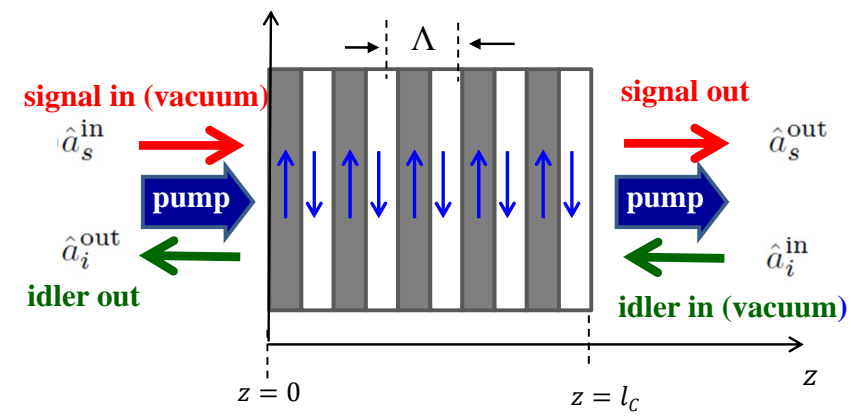

FIG. 1: (Color online) Geometry of the counterpropagating down-conversion (see text).

Counterpropagating PDC presents unique features, as the presence of a threshold for the pump intensity beyond which coherent parametric oscillations take place [11, thereby the name Mirrorless Optical parametric Oscillator $(\mathrm{MOPO})$ [8. In a related work [12, we study the quantum correlation of counter-propagating twin beams close to the threshold; here, instead, we focus on the regime of spontaneous photon pairs production, well below threshold, and analyse the temporal quantum properties of counterpropagating twin photons generated in a purely collinear 
configuration.

A second peculiar feature of the MOPO is the narrow spectral bandwidth of emission (the backward propagating wave can be even more monochromatic than the pump laser [8]). In the quantum domain, as pointed out in [14], counterpropagating SPDC can generate highly monochromatic photon pairs in an almost separable state, which makes it a promising source of high-purity heralded single photons.

In this work we provide a detailed theoretical analysis of the effects of the spectral properties of the pump laser on the degree of entanglement of the state, identify the physical conditions under which the state may become separable, and provide a consistent interpretation of the transition from an entangled to a separable state.

In particular we will show that the system dynamics is governed by two well separated time scales: a long one, related to the temporal separation of counterpropagating waves, which is on the order of the transit time of light along the crystal (tens of picoseconds), and a short one related to the temporal separation of co-propagating waves due to their different group velocities (order 1ps or smaller). When the pump pulse duration is intermediate between the two scales, we will show that the two-photon state becomes separable, and remains separable for a wide range of pump durations, whilst it has a high degree of entanglement in the two opposite limits. Notice that such a difference of time scales occurs naturally in the counterpropagating configuration, for basically any kind of material and tuning condition. This is quite different from the co-propagating case where separability of the state requires special operational points 26 .

In addition, we shall investigate the coherence properties of the SPDC photons taken individually, showing a transition from a symmetric state, for a long pump pulse, to a highly asymmetric state for a short pump pulse. In particular, in the regime where the state is separable, the spectrum of the signal turns out to reproduce the spectrum of the co-propagating pump laser, while that of the backward propagating idler is entirely determined by the crystal properties.

The paper is organized as follows: Section I introduces a quantum model for counterpropagating PDC; Sec. II characterizes the quantum correlation of twin photons in the spectral domain, while Sec III is devoted to their coherence properties. Sec IV quantifies the degree of entanglement of the state via the Schmidt number. Finally Sec $\mathrm{V}$ gives an interpretation of the transition from entanglement to separability by analysing the correlation in the temporal domain.

\section{THE MODEL}

The starting point of our analysis are the equations describing the propagation of three interacting pump, signal and idler waves along a slab of periodically poled second order nonlinear crystal (Fig.1). We shall consider here only collinear propagation, either assuming that light is collected only at small propagation angles with respect to the pump, or because of a waveguiding configuration. In a crystal with periodic inversion of the nonlinear susceptibility, the momentum conservation in the three-wave interaction is replaced by a less restrictive conservation law (quasiphase matching) [17], which includes also the momenta $\frac{2 \pi}{\Lambda} m$ of the nonlinear lattice, where $\Lambda$ is the poling period (for example $m= \pm 1, \pm 3 \ldots$ for a simple poling). Since the effective nonlinearity is higher for lower orders, one usually tries to phase-match the first order $m= \pm 1$ interaction. The counter-propagating configuration, in which one wave (say the idler) is generated in the backward direction with respect to the pump laser (Fig.1), thus requires a poling period on the order of the pump wavelength, because the pump momentum needs to be almost entirely compensated by the grating momentum $k_{G}=\frac{2 \pi}{\Lambda}$. In these conditions, the central frequencies $\omega_{s}, \omega_{i}=\omega_{p}-\omega_{s}$ of the down-converted wavepackets are determined by quasi-phase matching at the central pump frequency $\omega_{p}$

$$
k_{0 s}-k_{0 i}=k_{0 p}-\frac{2 \pi}{\Lambda}
$$

where $k_{0 j}=n_{j}\left(\omega_{j}\right) \omega_{j} / c, j=s, i, p$ are the wave numbers at the three central frequencies. We shall mostly focus on the commonly realized type $\mathrm{O}$ interaction [8], where the three waves have the same polarization, but we leave the formalism quite general. Hence the subscript $\mathrm{j}$ in the wave number may refer to dispersion relations for either the ordinary or extraordinary wave, including thus type II or I PDC.

We than introduce the positive frequency part of field operators (with dimension of photon destruction operators) for the three wavepackets as

$$
\begin{aligned}
& \hat{A}_{s}(\Omega, z)=e^{+i k_{s}(\Omega) z} \hat{a}_{s}(\Omega, z), \\
& \hat{A}_{i}(\Omega, z)=e^{-i k_{i}(\Omega) z} \hat{a}_{i}(\Omega, z), \\
& \hat{A}_{p}(\Omega, z)=e^{+i k_{p}(\Omega) z} \hat{a}_{p}(\Omega, z),
\end{aligned}
$$


where capital $\Omega$ is a frequency offset from the carrier frequencies, and $k_{j}(\Omega)$ are the wave numbers at frequency $\omega_{j}+\Omega$. In this definition, the factors $e^{ \pm i k_{j}(\Omega) z}$ account for all the effects of the linear propagation along the medium. Hence the operators $\hat{a}_{j}$ have a slow variation along the crystal because they evolve only under the effects of the nonlinear interaction. Their coupled equations of propagation can thus be written as ( see [12] for a more detailed analysis)

$$
\begin{aligned}
\frac{\partial}{\partial z} \hat{a}_{s}(\Omega, z) & =\chi \int d \Omega^{\prime} \hat{a}_{p}\left(\Omega+\Omega^{\prime}, z\right) \hat{a}_{i}^{\dagger}\left(\Omega^{\prime}, z\right) e^{-i \mathcal{D}\left(\Omega, \Omega^{\prime}\right) z} \\
\frac{\partial}{\partial z} \hat{a}_{i}(\Omega, z) & =-\chi \int d \Omega^{\prime} \hat{a}_{p}\left(\Omega+\Omega^{\prime}, z\right) \hat{a}_{s}^{\dagger}\left(\Omega^{\prime}, z\right) e^{-i \mathcal{D}\left(\Omega^{\prime}, \Omega\right) z} \\
\frac{\partial}{\partial z} \hat{a}_{p}(\Omega, z) & =-\chi \int d \Omega^{\prime} \hat{a}_{s}\left(\Omega^{\prime}, z\right) \hat{a}_{i}\left(\Omega-\Omega^{\prime}, z\right) e^{i \mathcal{D}\left(\Omega, \Omega-\Omega^{\prime}\right) z}
\end{aligned}
$$

where $\chi$ is proportional to the effective second order susceptibility of the crystal, and only the first order terms \pm 1 in the Fourier espansion of the periodic nonlinear suceptibility have been retained (namely order -1 for signal and idler, order +1 for the pump). In these equations

$$
\mathcal{D}\left(\Omega, \Omega^{\prime}\right)=k_{s}(\Omega)-k_{i}\left(\Omega^{\prime}\right)-k_{p}\left(\Omega+\Omega^{\prime}\right)^{\prime}+\frac{2 \pi}{\Lambda}
$$

is the effective phase mismatch that rules the efficiency of each elementary down-conversion process, where a signal and an idler photon of frequencies $\omega_{s}+\Omega, \omega_{i}+\Omega^{\prime}$ are generated out of a pump photon of frequency $\omega_{p}+\Omega+\Omega^{\prime}$. Notice that, a part from the different form of the phase matching (4), the only formal difference with the usual co-propagating case (see e.g. [16]) is the minus sign appearing at r.h.s of (3) b for the counterpropagating idler. As we shall see in the following, however, this minus sign leads to very relevant physical differences.

\section{A. Low-gain limit}

In a parent work [12 we analyse these equations in a generic gain regime, including also the region close to the MOPO threshold. In this work we instead focus on the ultra-low gain regime, much below the MOPO threshold, where photons pairs are generated by purely spontaneous down-conversion. In this regime, the depletion of the pump beam can be neglected and the pump approximated by a constant c-number field, corresponding to the pump pulse at the crystal input face

$$
\hat{a}_{p}(\Omega, z) \rightarrow \alpha_{p}(\Omega, z) \approx \alpha_{p}(\Omega, z=0)
$$

The strength of the parametric coupling is then described by the dimensionless gain parameter

$$
g=\sqrt{2 \pi} \chi \alpha_{p}(t=0) l_{c}
$$

where $\alpha_{p}(t=0)$ is the peak value of the pump temporal profile. Notice that in the limit of a monochromatic pump [12, 13. $g=\pi / 2$ represents the threshold for the MOPO. Conversely, in the limit $g \ll 1$ Eqs. (3) can be solved perturbatively. Namely, we write the formal solution of (3), starting from the boundary conditions:

$$
\begin{aligned}
& \hat{a}_{s}(\Omega, z=0)=\hat{a}_{s}^{\text {in }}(\Omega) \\
& \hat{a}_{i}\left(\Omega, z=l_{c}\right)=\hat{a}_{i}^{\text {in }}(\Omega)
\end{aligned}
$$

determined by the input signal and idler fields, entering the crystal from the left face of at $z=0$ and from the right face at $z=l_{c}$, respectively (Fig.1). By solving iteratively, a perturbative seriers of powers of $g$ is obtained. By keeping only the first order terms in $g \ll 1$, one obtains a Boguliobov linear trasformation that links the output to the input operators:

$$
\begin{aligned}
\hat{a}_{s}^{\text {out }}\left(\Omega_{s}\right) & =\hat{a}_{s}\left(\Omega_{s}, z=l_{c}\right) \\
& =\hat{a}_{s}^{\text {in }}\left(\Omega_{s}\right)+\int d \Omega_{i} \psi\left(\Omega_{s}, \Omega_{i}\right) \hat{a}_{i}^{\text {in } \dagger}\left(\Omega_{i}\right), \\
\hat{a}_{i}^{\text {out }}\left(\Omega_{i}\right) & =\hat{a}_{i}\left(\Omega_{s}, z=0\right) \\
& =\hat{a}_{i}^{\text {in }}\left(\Omega_{i}\right)+\int d \Omega_{s} \psi\left(\Omega_{s}, \Omega_{i}\right) \hat{a}_{s}^{\text {in } \dagger}\left(\Omega_{s}\right) .
\end{aligned}
$$


with the biphoton amplitude given by

$$
\begin{aligned}
\psi\left(\Omega_{s}, \Omega_{i}\right) & =\frac{g}{\sqrt{2 \pi}} \tilde{\alpha}_{p}\left(\Omega_{s}+\Omega_{i}\right) \\
& \times \operatorname{sinc}\left[\frac{\mathcal{D}\left(\Omega_{s}, \Omega_{i}\right) l_{c}}{2}\right] e^{-i \frac{\mathcal{D}\left(\Omega_{s}, \Omega_{i}\right) l_{c}}{2}}
\end{aligned}
$$

where

$$
\tilde{\alpha}_{p}(\Omega)=\int \frac{d t}{\sqrt{2 \pi}} e^{i \Omega t} \frac{\alpha(t)}{\alpha_{p}(t=0)}
$$

is the Fourier profile of the pump pulse at the crystal input face, normalized to its temporal peak value. Notice that Eqs. (9) define a unitary tranformation only up to first order in $g$. In the following, the input signal and idler field at the left and right end faces of the crystal will be taken in the vacuum state.

It is worth remarking that the quantum field formalism here employed can be replaced by an equivalent state formalism (see e.g [4 for more details) where the state evolves instead of the field operators. By applying the trasformation $(9)$ to the input vacuum state, one obtains at the output the usual state

$$
|\phi\rangle_{\mathrm{OUT}}=|0\rangle+\frac{1}{2} \int d \Omega_{s} d \Omega_{i} \psi\left(\Omega_{s}, \Omega_{i}\right) \hat{a}_{s}^{\dagger}\left(\Omega_{s}\right) \hat{a}_{i}^{\dagger}\left(\Omega_{i}\right)|0\rangle
$$

describing the superposition of the vacuum state $|0\rangle$ and of a two-photon state, where the photon pair can be generated in any of the Fourier modes $\Omega_{s}, \Omega_{i}$ with probability amplitude $\psi\left(\Omega_{s}, \Omega_{i}\right)$. In this respect, the formalism used here is equivalent to the one employed in [14, 15].

\section{SPECTRAL BIPHOTON CORRELATION}

This section is devoted to the analysis of the biphotonic correlation in the spectral domain. Precisely, we focus on the probability amplitude $\left\langle\hat{A}_{s}^{\text {out }}\left(\Omega_{s}\right) \hat{A}_{i}^{\text {out }}\left(\Omega_{i}\right)\right\rangle$ of finding a pair of photons at frequencies $\Omega_{s}, \Omega_{i}$ at the crystal output faces. Using the input-output relations $(9)$ and the definitions $(2)$ :

$$
\left\langle\hat{A}_{s}^{\text {out }}\left(\Omega_{s}\right) \hat{A}_{i}^{\text {out }}\left(\Omega_{i}\right)\right\rangle=e^{i k_{s}\left(\Omega_{s}\right) l_{c}} \psi\left(\Omega_{s}, \Omega_{i}\right) .
$$

with $\psi$ given by Eq. (10). As usual, the biphoton correlation is the product of two terms: i) the pump spectral amplitude $\tilde{\alpha}_{p}\left(\Omega_{s}+\Omega_{i}\right)$, reflecting the energy conservation in the microscopic process, and ii) the phase matching function $\operatorname{sinc}\left(\mathcal{D} l_{c} / 2\right) e^{-i \mathcal{D} l_{c} / 2}$, reflecting the generalized momentum conservation. Concerning the latter, we can expand $\mathcal{D}\left(\Omega_{s}, \Omega_{i}\right)$ in Eq. (4) in power series of the frequency shifts from the carriers. Down-conversion spectra are typically narrow [8, 9], as will become also clear in the following, so that one is allowed to retain only terms up to first order

$$
\begin{aligned}
\mathcal{D}\left(\Omega_{s}, \Omega_{i}\right) \frac{l_{c}}{2} & \approx \frac{l_{c}}{2}\left[\left(k_{s}^{\prime}-k_{p}^{\prime}\right) \Omega_{s}-\left(k_{i}^{\prime}+k_{p}^{\prime}\right) \Omega_{i}\right] \\
& =-\left(\frac{\Omega_{s}}{\Omega_{\mathrm{gvm}}}+\frac{\Omega_{i}}{\Omega_{\mathrm{gvs}}^{\prime}}\right),
\end{aligned}
$$

where the zero order term vanishes because of Eq. [1], and $k_{i}^{\prime}=d k_{j} /\left.d \omega\right|_{\omega=\omega_{j}}, j=s, i, p$. We thus see the appearence of the two characteristic temporal scales:

$$
\begin{aligned}
\tau_{\mathrm{gvm}} & :=\Omega_{\mathrm{gvm}}{ }^{-1}=\frac{1}{2}\left[\frac{l_{c}}{v_{g p}}-\frac{l_{c}}{v_{g s}}\right] \\
\tau_{\mathrm{gvs}}^{\prime} & :=\Omega_{\mathrm{gvs}}^{\prime}{ }^{-1}=\frac{1}{2}\left[\frac{l_{c}}{v_{g p}}+\frac{l_{c}}{v_{g i}}\right],
\end{aligned}
$$

where $v_{g i}=1 / k_{i}^{\prime}$ are the group velocities of the three wavepackets at the central frequencies. The first scale [Eq. [16]]describes the " small" temporal separation between the co-propagating waves due to their group velocity mismatch (GVM) . The second one [Eq. [17]] accounts for the "large"temporal separation of the counter-propagating pump and idler waves, which is ruled by the time needed by the pulse centers to cross the crystal. Closely related,

$$
\tau_{\mathrm{gvs}}=\Omega_{\mathrm{gvs}}{ }^{-1}=\frac{1}{2}\left[\frac{l_{c}}{v_{g s}}+\frac{l_{c}}{v_{g i}}\right]
$$


describes the characteristic temporal separation between the arrival times of an idler and a signal photon at their exit faces. Clearly, since group velocities are close, $\tau_{\mathrm{gvs}} \approx \tau_{\mathrm{gvs}}^{\prime}$, while $\tau_{\mathrm{gvm}} \ll \tau_{\mathrm{gvs}}^{\prime}, \tau_{\mathrm{gvs}}$, and

$$
\eta=\frac{\tau_{\mathrm{gvm}}}{\tau_{\mathrm{gvs}}^{\prime}}=\frac{\Omega_{\mathrm{gvs}}^{\prime}}{\Omega_{\mathrm{gvm}}} \ll 1 .
$$

Therefore, the phase matching has two well separated scales of variation: as a function of the signal frequency it decays on the broad bandwidth $\Omega_{\mathrm{gvm}}$, while as a function of the idler frequency it decays on the narrow bandwidth $\Omega_{\text {gvs }}^{\prime}$. Plots of the parameter $\eta$, for periodically poled KTP (potassium titanyl phosphate) and $\mathrm{LiNbO}_{3}$ (lithium niobate),

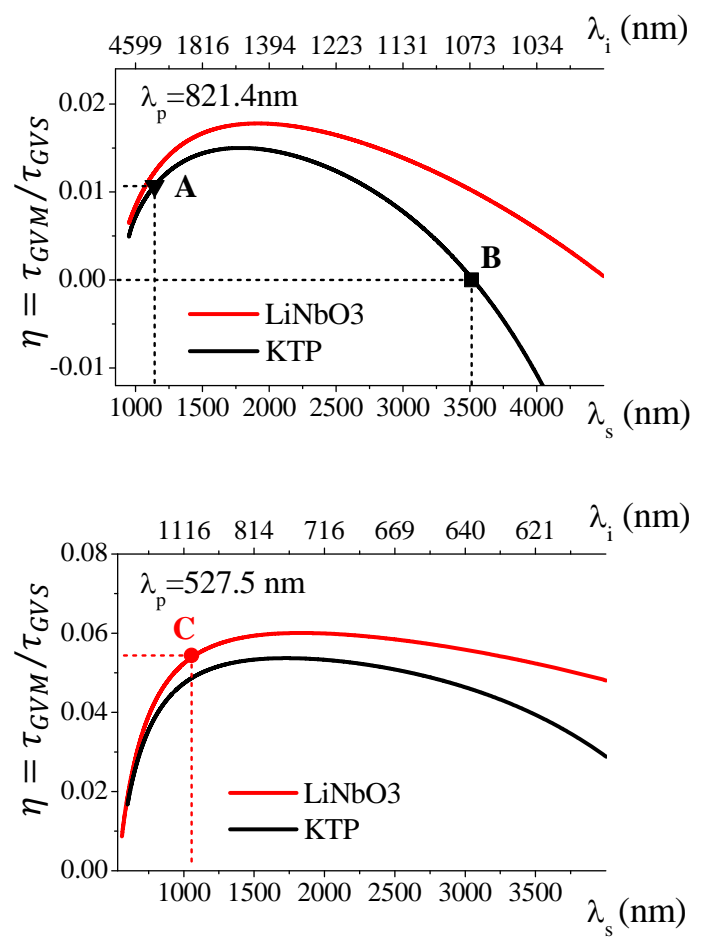

FIG. 2: (Color online) Ratio $\eta=\tau_{\text {gvm }} / \tau_{\text {gvs }}^{\prime}$ for periodically poled KTP and $\mathrm{LiNbO}_{3}$, pumped in the infrared or visible, for type $0 e \rightarrow e e$ down-conversion. Point $\mathbf{A}$ is KTP pumped at $\lambda_{p}=821 \mathrm{~nm}$, with $\Lambda_{\text {pol }}=800 \mathrm{~nm}, \lambda_{s}=1141 \mathrm{~nm}, \lambda_{i}=2932 \mathrm{~nm}$, corresponding to the experiment in [8]; B is the zero GVM point for the KTP at $\lambda_{p}=821 \mathrm{~nm}$, corresponding to $\Lambda_{p o l}=290 \mathrm{~nm}$, $\lambda_{s}=3523 \mathrm{~nm}, \lambda_{i}=1071 \mathrm{~nm}$. $\mathbf{C}$ is a $\mathrm{LiNbO}_{3}$ slab pumped at $\lambda_{p}=527.5 \mathrm{~nm}$, for degenerate $\mathrm{PDC}$ at $\lambda_{s}=\lambda_{i}=1055 \mathrm{~nm}$, with $\Lambda_{\text {pol }}=236 \mathrm{~nm}$.

are shown in Fig 2, where $\mathbf{A}, \mathbf{B}, \mathbf{C}$ are the points that will be used as examples in the following.

Finally, a third relevant scale is the pump spectral bandwidth. For a coherent Gaussian pump $\alpha_{p}(t)=$ $\alpha_{p}(0) \exp -\frac{t^{2}}{2 \tau_{p}^{2}}$, the pulse duration $\tau_{p}$ is the inverse of the bandwidth

$$
\tau_{\mathrm{p}}=\frac{1}{\Delta \Omega_{\mathrm{p}}}
$$

Depending on the pump bandwidth relative to the spectral scales of phase matching, different physical situations arise. The three relevant possibilities, depicted in Fig, 3 , will be studied separately in the following.

\section{i) Limit of a CW pump:}

We assume a narrowband pump pulse, such that

$$
\tau_{p} \gg \tau_{\text {gvs }}^{\prime} \gg \tau_{\text {gvm }}, \text { or } \Delta \Omega_{\mathrm{p}} \ll \Omega_{\text {gvs }}^{\prime} \ll \Omega_{\text {gvm }} .
$$

This limit corresponds to a pump pulse that in the $z$ direction is much longer than the crystal slab, and for a crystal of some mm length requires a pulse duration of hundreds of picoseconds or longer. In this limit the pump spectral profile 
(a) $\tau_{\mathrm{p}}=353 \mathrm{ps}$

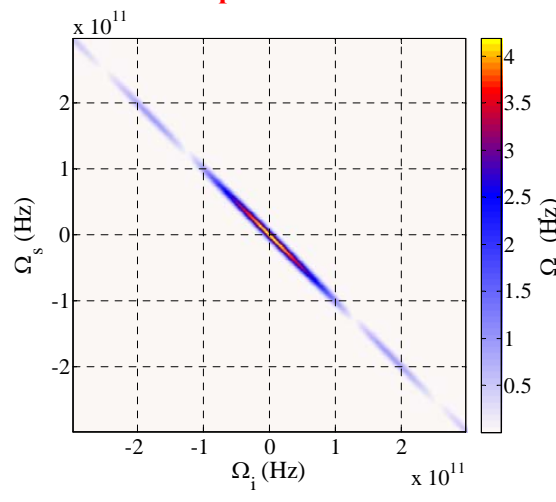

(b) $\tau_{\mathrm{p}}=1.1 \mathrm{ps}$

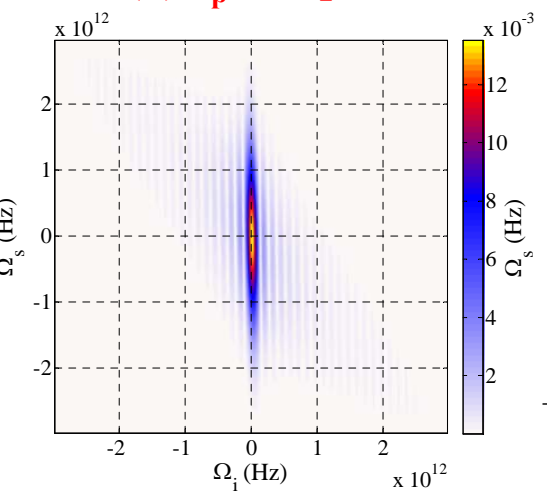

(c) $\tau_{\mathrm{p}}=\mathbf{0 . 0 3} \mathrm{ps}$

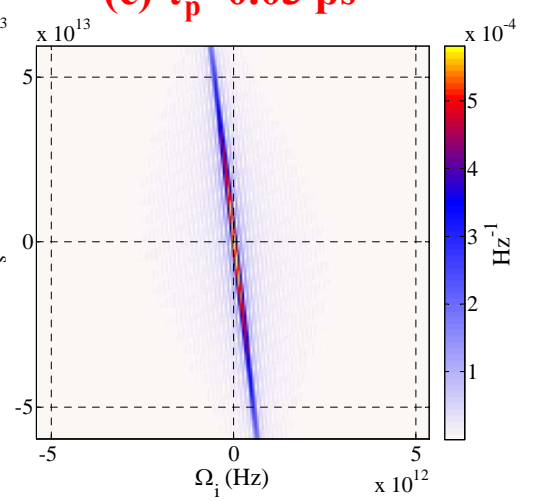

FIG. 3: (Color online) Biphoton correlation $|\psi|$ [Eq. 10$]$ in the plane $\left(\Omega_{i}, \Omega_{s}\right)$, in various pumping regimes. Example of a $4 \mathrm{~mm}$ PPKTP, pumped at $821.4 \mathrm{~nm}$, corresponding to the point $\mathbf{A}$ in Fig 2 , with $\tau_{\text {gvs }}^{\prime}=25.2 \mathrm{ps}, \tau_{\mathrm{gvm}}=0.27 \mathrm{ps}$. (a) Quasi CW pump pulse $\tau_{\mathrm{p}}=253 \mathrm{ps}$. (b) Intermediate pump pulse $\tau_{p}=1.1 \mathrm{ps}$. (c) Ultrashort pump $\tau_{\mathrm{p}}=0.03$ ps . Note the different scales of the plots $10^{11} \rightarrow 10^{13} \mathrm{~Hz}$.

$\tilde{\alpha}_{p}\left(\Omega_{s}+\Omega_{i}\right)$ is much narrower than the phase matching bandwidths, and the geometry of the correlation is dominated by energy conservation, which requires that the twins are generated at symmetric frequencies $\Omega_{s}+\Omega_{i}=\Omega_{p} \approx 0$. As a consequence, the biphoton correlation 10 has a sharp maximum along the diagonal $\Omega_{s}=-\Omega_{i}$, as shown by Fig 3 a Indeed, as derived in the Appendix A1, in this limit the correlation is well approximated by :

$$
\begin{aligned}
\psi\left(\Omega_{s}, \Omega_{i}\right) & \simeq \frac{g}{\sqrt{2 \pi}} \tilde{\alpha}_{p}\left(\Omega_{s}+\Omega_{i}\right) \operatorname{sinc}\left(\frac{\Omega_{s}}{\Omega_{\mathrm{gvs}}}\right) e^{-i \frac{\Omega_{s}}{\Omega_{\mathrm{gvs}}}} \\
& \simeq \frac{g}{\sqrt{2 \pi}} \tilde{\alpha}_{p}\left(\Omega_{s}+\Omega_{i}\right) \operatorname{sinc}\left(\frac{\Omega_{i}}{\Omega_{\mathrm{gvs}}}\right) e^{i \frac{\Omega_{i}}{\Omega_{\mathrm{gvs}}}}
\end{aligned}
$$

\section{ii) Limit of an ultrashort pump pulse:}

We consider here the limit:

$$
\tau_{p} \ll \tau_{\mathrm{gvm}}, \tau_{\mathrm{gvs}}^{\prime}, \text { or } \Delta \Omega_{\mathrm{p}} \gg \Omega_{\mathrm{gvm}}, \Omega_{\mathrm{gvs}}^{\prime},
$$

where the pump pulse is not only shorter than the crystal length, but also shorter than the average separation between the pump and signal wavepackets due their GVM. In our examples this corresponds to duration shorter than 100 fs. In these conditions, the pump spectral profile $\tilde{\alpha}_{p}\left(\Omega_{s}+\Omega_{i}\right)$ decays slowly with respect to sinc $\mathcal{D}\left(\Omega_{s}, \Omega_{i}\right) l_{c} / 2$, so that the geometry of the biphoton correlation is dominated by the phase matching, i.e. by the momentum conservation. As a result (Fig 3 ;) the biphoton correlation takes the approximated form

$$
\begin{aligned}
\psi\left(\Omega_{s}, \Omega_{i}\right) & \simeq \frac{g}{\sqrt{2 \pi}} \tilde{\alpha}_{p}\left[\Omega_{s}(1-\eta)\right] \operatorname{sinc}\left(\frac{\Omega_{s}}{\Omega_{\mathrm{gvm}}}+\frac{\Omega_{i}}{\Omega_{\mathrm{gvs}}^{\prime}}\right) e^{i\left(\frac{\Omega_{s}}{\Omega_{\mathrm{gvm}}}+\frac{\Omega_{i}}{\Omega_{\mathrm{gvs}}}\right)} \\
& \simeq \frac{g}{\sqrt{2 \pi}} \tilde{\alpha}_{p}\left[-\Omega_{i} \frac{1-\eta}{\eta}\right] \operatorname{sinc}\left(\frac{\Omega_{s}}{\Omega_{\mathrm{gvm}}}+\frac{\Omega_{i}}{\Omega_{\mathrm{gvs}}^{\prime}}\right) e^{i\left(\frac{\Omega_{s}}{\Omega_{\mathrm{gvm}}}+\frac{\Omega_{i}}{\Omega_{\mathrm{gvs}}^{\prime}}\right)}
\end{aligned}
$$

When plotted in the plane $\left(\Omega_{i}, \Omega_{s}\right)$, the function shows a sharp maximum along the line

$$
\Omega_{s}=-\Omega_{i} \frac{\Omega_{\mathrm{gvm}}}{\Omega_{\mathrm{gvs}}^{\prime}}
$$

where phase matching occurs (see Eq. 15 ), and very asymmetric spectral properties of the signal -idler photons.

iii) Intermediate pump pulse:

The intermediate case, where

$$
\tau_{\mathrm{gvs}}^{\prime} \gg \tau_{p} \gg \tau_{\mathrm{gvm}}, \text { or } \Omega_{\mathrm{gvs}}^{\prime} \ll \Delta \Omega_{\mathrm{p}} \ll \Omega_{\mathrm{gvm}}
$$


is the most peculiar one, because the biphoton correlation may approach a separable function of $\Omega_{s}, \Omega_{i}($ Fig $3 \mathrm{~b})$. First of all, we remark that the limit 28 is strictly realized only for $\eta=\tau_{\text {gvm }} / \tau_{\text {gvs }}^{\prime} \rightarrow 0$, i.e for a vanishing group velocity mismatch between the pump and the signal. This condition is favourable to separabilty, because as $\eta \rightarrow 0$ the phase matching function tends to become a stripe parallel to the $\Omega_{s}$ axis (see Eq. (27) ), but it is not a sufficient one, because of the role of the pump profile in Eq. 10. However, provided that the the pump spectrum satisfies the intermediate limit (28), it can be demonstrated (Appendix A1) that the the biphoton amplitude (10) approaches the factorized form:

$$
\psi\left(\Omega_{s}, \Omega_{i}\right) \rightarrow \frac{g}{\sqrt{2 \pi}} \tilde{\alpha}_{p}\left(\Omega_{s}\right) e^{i \frac{\Omega_{s}}{\Omega_{\mathrm{gvm}}}} \times \operatorname{sinc}\left(\frac{\Omega_{i}}{\Omega_{\mathrm{gvs}}}\right) e^{i \frac{\Omega_{i}}{\Omega_{\mathrm{gvs}}^{\prime}}},
$$

i.e. it becomes the product of a function of $\Omega_{s}$, reproducing the pump profile, and a function of $\Omega_{i}$, corresponding to the phase matching profile. This describes a nonentangled biphoton state, with the signal photon generated in the same spectro- temporal mode as the pump, while the spectral mode of the idler is dictated by the phase matching "sinc" function of width $\Omega_{\text {gvs }}$.

This qualitative picture will be confirmed by the evaluation of the Schmidt number in Sec. IV, and will be further interpreted and discussed in the light of the temporal correlation of biphotons described in Sec.V

\section{SPECTRAL COHERENCE OF COUNTERPROPAGATING PHOTONS}

This section is devoted to the marginal statistics of individual signal and idler photons. The focus is on their spectral coherence properties, studied by means of the first order coherence functions

$$
\begin{aligned}
& G_{s}^{(1)}\left(\Omega, \Omega^{\prime}\right)=e^{-i\left[k_{s}\left(\Omega^{\prime}\right)-k_{s}(\Omega)\right] l_{c}}\left\langle\hat{A}_{s}^{\dagger \mathrm{o} u t}(\Omega) \hat{A}_{s}^{\mathrm{ou} t}\left(\Omega^{\prime}\right)\right\rangle \\
& G_{i}^{(1)}\left(\Omega, \Omega^{\prime}\right)=\left\langle\hat{A}_{i}^{\dagger \mathrm{ou} t}(\Omega) \hat{A}_{i}^{\mathrm{out}}\left(\Omega^{\prime}\right)\right\rangle
\end{aligned}
$$

(where a propagation phase factor is present in the first definition just for convenience of notation). From the input-output relations $\sqrt{9}$ one has:

$$
\begin{aligned}
G_{s}^{(1)}\left(\Omega_{s}, \Omega_{s}^{\prime}\right) & =\int d \Omega_{i} \psi^{*}\left(\Omega_{s}, \Omega_{i}\right) \psi\left(\Omega_{s}^{\prime}, \Omega_{i}\right), \\
G_{i}^{(1)}\left(\Omega_{i}, \Omega_{i}^{\prime}\right) & =\int d \Omega_{s} \psi^{*}\left(\Omega_{s}, \Omega_{i}\right) \psi\left(\Omega_{s}, \Omega_{i}^{\prime}\right) .
\end{aligned}
$$

i.e. the coherence functions are convolution integrals over the biphoton amplitude $\psi$, given by Eq 10 . The knowledge of the $G_{j}^{(1)}$ is sufficient to determine all the statistical properties of the marginal distributions. For example, the autocorrelation of the light intensities $\hat{I}_{j}=\hat{A}_{j}^{\dagger} \hat{A}_{j}$ is given by

$$
\left\langle\hat{I}_{j}(\Omega) \hat{I}_{j}\left(\Omega^{\prime}\right)\right\rangle=\delta\left(\Omega-\Omega^{\prime}\right)\left\langle\hat{I}_{j}(\Omega)\right\rangle+\left\langle\hat{I}_{j}(\Omega)\right\rangle\left\langle\hat{I}_{j}\left(\Omega^{\prime}\right)\right\rangle+\left|G_{j}^{(1)}\left(\Omega, \Omega^{\prime}\right)\right|^{2},
$$

where $\left\langle\hat{I}_{j}(\Omega)\right\rangle=G_{j}^{(1)}(\Omega, \Omega)$. This relation, which is a consequence of the factorization theorem of Gaussian moments, is typical of thermal-like statistics. As a matter of fact, the marginal distributions of the output signal-idler light are thermal-like Gaussian, when there is vacuum at the input. In the low-gain regime considered here, the dominant term is the first one, i.e. the "shot-noise" term $\delta$-correlated in frequencies. Therefore, as well known in this regime the statistics of photon counts in each arm is Poissonian.

On the other side, the convolution integrals in Eqs. [31, , 32 indicate that an autocorrelation of spectral fluctuations inside each individual signal or idler wave exists because of second order processes, that involve the probability amplitudes of generating at two pairs of photons.

In the following we shall illustrate the three relevant cases. The coherence functions will be evaluated both numerically (Fig, 4) and analytically. In the first case, the complete Sellmeier relations [18] will be used to compute the integrals in 31 , 32 , while the linear approximation for phase matching will be exploited to derive approximated analytical formulas.

\section{i) Limit of a CW pump:}

Column (a) of Fig 4 shows an example of the signal and idler coherence functions in the plane $\left(\Omega, \Omega^{\prime}\right)$, numerically computed in the case of a long pump pulse $\tau_{p} \simeq 14 \tau_{\mathrm{gvs}}^{\prime}$. 

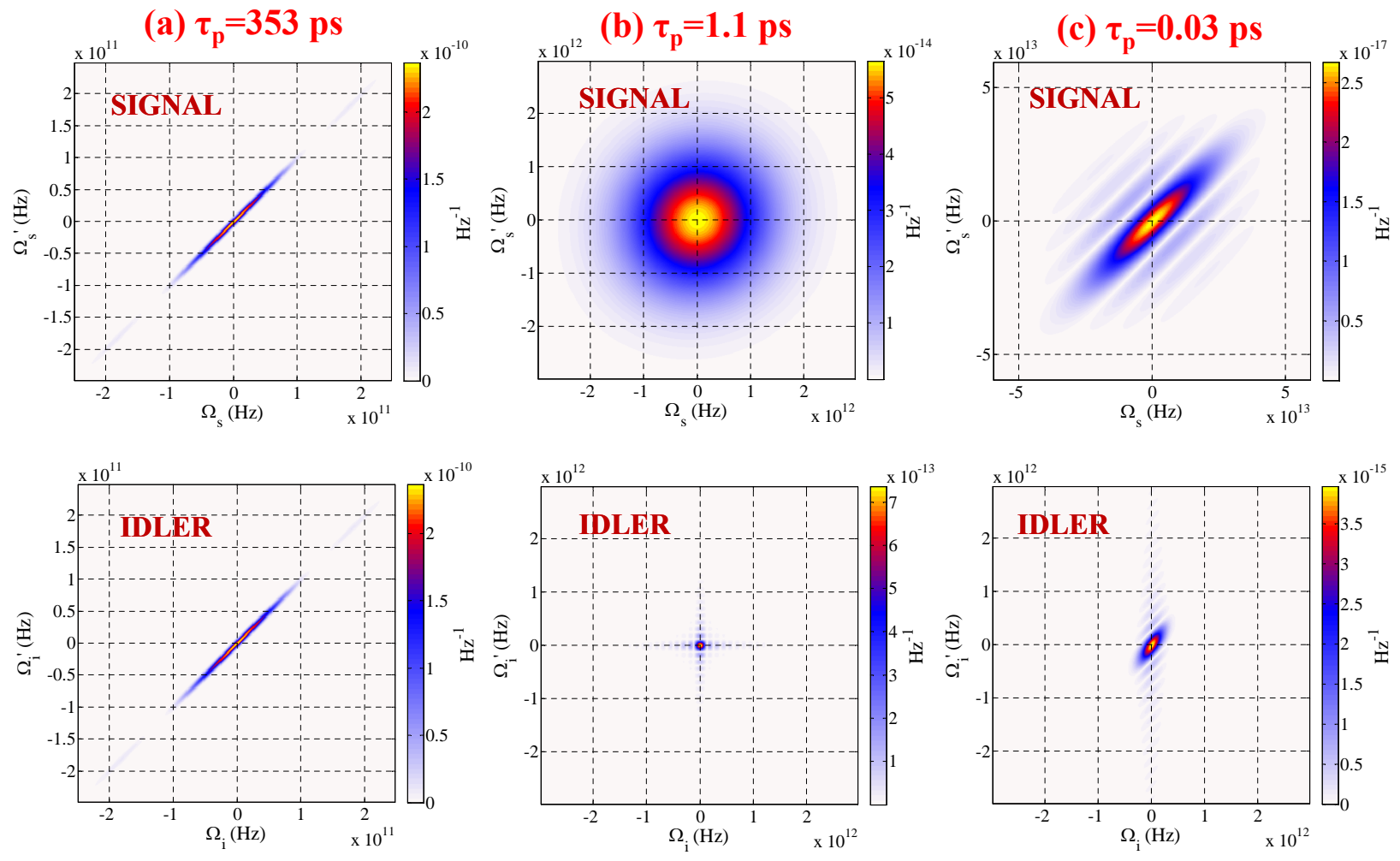

FIG. 4: (Color online) The coherence functions $\left|G\left(\Omega, \Omega^{\prime}\right)\right|$ of the forward signal and backward propagating idler are plotted in the upper and lower row, respectively, for different pumping regime. Column (a) Quasi CW pump pulse $\tau_{\mathrm{p}}=353 \mathrm{ps}$. (b) Intermediate pump pulse $\tau_{p}=1.1 \mathrm{ps}$. (c) Ultrashort pump $\tau_{\mathrm{p}}=0.03$ ps. Same KTP crystal slab as in Fig 3 (point $\mathbf{A}$ in Fig. 2), with $\tau_{\mathrm{gvs}}^{\prime}=25.2 \mathrm{ps}, \tau_{\mathrm{gvm}}=0.27 \mathrm{ps}$. Note the different scales in the panels

In the limit $\tau_{p} \gg \tau_{\text {gvs }}^{\prime}$, approximated expressions for the coherence functions can be calculated by inserting the formulas for the biphoton correlation (22) and 23), valid in this limit, into Eqs. (31) and (32), respectively, and performing the simple integrals. After some passages:

$$
\begin{aligned}
G_{i}^{(1)}\left(\Omega, \Omega^{\prime}\right) & \simeq G_{s}^{(1)}\left(\Omega, \Omega^{\prime}\right) \\
& \stackrel{\tau_{\mathrm{p}} \gg \tau_{\text {gvs }}^{\prime}}{\longrightarrow} \tilde{\mathcal{I}}_{p}\left(\Omega^{\prime}-\Omega\right) g^{2} \operatorname{sinc}^{2}\left(\frac{\Omega}{\Omega_{\mathrm{gvs}}}\right)
\end{aligned}
$$

where $\tilde{\mathcal{I}}_{p}(\Omega)=\int \frac{d t}{2 \pi} e^{i \Omega t}\left|\alpha_{p}(t) / \alpha_{p}(0)\right|^{2}$ is the Fourier transform of the pump intensity profile. These approximated formulas have been checked with the numerical results and show an excellent match. These results may be considered the more refined version of the much simpler CW model analysed in [12, 13, with the narrow peak $\tilde{\mathcal{I}}_{p}\left(\Omega^{\prime}-\Omega\right)$ being the finite counterpart of the singular Dirac $\delta$ appearing in the strictly CW pump model [12].

For a quasi-CW pump the counter-propagating signal and idler photons are predicted to have identical spectral coherence properties. In particular, by looking at the $G^{(1)}$ functions along the diagonal $\Omega^{\prime}=\Omega$ we see that their $\operatorname{spectra}\left\langle\hat{I}_{j}(\Omega)\right\rangle=\left\langle\hat{A}_{j}^{\dagger}(\Omega) \hat{A}_{j}(\Omega)\right\rangle$

$$
\left\langle\hat{I}_{s}(\Omega)\right\rangle=\left\langle\hat{I}_{i}(\Omega)\right\rangle \simeq \frac{g^{2} \tau_{p}}{\sqrt{2 \pi}} \operatorname{sinc}^{2}\left(\frac{\Omega}{\Omega_{\mathrm{gvs}}}\right),
$$

are identical and entirely determined by the narrow bandwidth of phase matching $\Omega_{\mathrm{gvs}}$. This bandwidth is in turn the inverse of the characteristic separation $\tau_{\text {gvs }}$ between the arrival time of an idler and a signal photon at their crystal end faces, which rouhgly corresponds to the long transit time of light along the crystal slab, because they propagate in opposite directions. As already noticed in [13], and as will be further discussed in Se(V this is clearly a big difference with the copropagating case. There, the temporal uncertainty between the arrival times of the idler and signal photon is short, because determined at most by the group velocity dispersion or mismatch, which results 
in the huge down-conversion bandwidths that characterize the standard co-propagating configuration.

On the other side, when studied as a function of $\Omega^{\prime}-\Omega$ the $G^{(1)}$ gives the characteristic size of spectral fluctuations, i.e. the spectral coherence length. This is determined by the pump bandwidth, more precisely by the width $\sqrt{2} \Delta \Omega_{p}$ of $\tilde{\mathcal{I}}_{p}\left(\Omega^{\prime}-\Omega\right)$, which is much narrower than the spectral bandwidths $\Omega_{\mathrm{gvs}}$. We can heuristically estimate the number of modes by counting the number of coherence length contained in the spectrum: therefore, for such a long pulse we expect each signal and idler photon to be generated in a highly incoherent and multimode state, with the number of modes $\propto \frac{\Omega_{\mathrm{gvs}}}{\Delta \Omega_{p}}=\frac{\tau_{\mathrm{p}}}{\tau_{\mathrm{gvs}}}$.

\section{ii) Ultrashort pump pulse:}

When the pump pulse shorten below the transit time $\tau_{\text {gvs }}^{\prime}$ along the crystal slab, the spectral properties of the counterpropagating idler and signal change drastically, becoming strongly asymmetric. First we consider the case of an ultrashort pulse, $\tau_{\mathrm{p}} \ll \tau_{\mathrm{gvm}}$ (i.e. such that pump and the signal tend to split apart during propagation). The asymmetry between the forward and backward propagating photons can be clearly appreciated in the third column of Fig. 4. which plots their coherence functions for $\tau_{p} \approx 0.1 \tau_{\mathrm{gvm}}$.

Approximated expressions for the coherence functions are derived also in this case, by using the limit behaviour of the biphoton correlation described by Eqs. 25) and (26). With some calculations:

$$
G_{s}^{(1)}\left(\Omega, \Omega^{\prime}\right) \stackrel{\tau_{\mathrm{p}} \ll \tau_{\mathrm{gvm}}}{\longrightarrow} \frac{g^{2} \Omega_{\mathrm{gvs}}^{\prime}}{2}\left|\tilde{\alpha}_{p}[\Omega(1-\eta)]\right|^{2} \operatorname{sinc}\left(\frac{\Omega^{\prime}-\Omega}{\Omega_{\mathrm{gvm}}}\right) e^{-i\left(\frac{\Omega^{\prime}-\Omega}{\Omega_{\mathrm{gvm}}}\right)}
$$

This formula predicts that the spectrum of the forward propagating signal

$$
\left\langle\hat{I}_{s}(\Omega)\right\rangle=\frac{g^{2} \Omega_{\mathrm{gvs}}^{\prime}}{2}\left|\tilde{\alpha}_{p}[\Omega(1-\eta)]\right|^{2}
$$

is a replica of the pump spectrum with a scale factor $\frac{1}{1-\eta}=\frac{k_{p}^{\prime}+k_{i}^{\prime}}{k_{i}^{\prime}+k_{s}^{\prime}}$ on the order unity. The coherence length of the signal (the characteristic size of spectral fluctuations) is instead determined by the width of the narrower sinc function, $l_{c o h, s} \approx \Omega_{\mathrm{gvm}}$. From this picture we thus expect that the signal photon, when detected independently from its twin, is in a incoherent multimode state, with the number of modes $\propto \frac{\Delta \Omega_{p}}{(1-\eta) \Omega_{\mathrm{gvm}}}$.

In a similar way, for the idler photon we get:

$$
G_{i}^{(1)}\left(\Omega, \Omega^{\prime}\right) \stackrel{\tau_{\mathrm{p}} \ll \tau_{\mathrm{gvm}}}{\longrightarrow} \frac{g^{2} \Omega_{\mathrm{gvm}}}{2}\left|\tilde{\alpha}_{p}\left[-\Omega \frac{1-\eta}{\eta}\right]\right|^{2} \times \operatorname{sinc}\left(\frac{\Omega^{\prime}-\Omega}{\Omega_{\mathrm{gvs}}^{\prime}}\right) e^{-i\left(\frac{\Omega^{\prime}-\Omega}{\Omega_{\mathrm{gvs}}}\right)}
$$

This formula predicts an idler bandwidth much narrower than the pump, precisely it predicts that the idler spectrum follows the pump spectrum with a scale factor $\frac{\eta}{1-\eta}=\frac{k_{p}^{\prime}-k_{s}^{\prime}}{k_{i}^{\prime}+k_{s}^{\prime}} \ll 1$. The coherence length of the idler is $l_{\text {coh }, i} \approx \Omega_{\mathrm{gvs}}^{\prime}$, so that the number of temporal modes is predicted to scale as $\frac{\eta \Delta \Omega_{p}}{(1-\eta) \Omega_{\mathrm{gvs}}^{\prime}}=\frac{\Delta \Omega_{p}}{(1-\eta) \Omega_{\mathrm{gvm}}}$, which is the same number as for the signal (as it must be because the signal and idler are the two members of the same entangled state, and their reduced states must exhibit the same Schmidt dimensionality, see next section) .

Notice that this particular scaling of the bandwidths of the forward and backward propagating waves with the pump bandwidth is well known in the literature concerning the MOPO. There, the same scaling factors, $\frac{k_{p}^{\prime}+k_{i}^{\prime}}{k_{i}^{\prime}+k_{s}^{\prime}}$ for the forward-propagating signal and $\frac{k_{p}^{\prime}-k_{s}^{\prime}}{k_{i}^{\prime}+k_{s}^{\prime}}$ for the backward propagating idler, are predicted to occurr [8, 9], by using arguments based on the phase-matching characteristic of the process. Here, however, the analysis concerns the quantum properties of the single photons generated well below the MOPO threshold. Moreover, at difference with the classical analysis in [8, such a scaling with the pump spectrum is predicted only in rather extreme conditions, corresponding to an ultrashort pump pulse $\tau_{p} \ll \tau_{\mathrm{gvm}}$. Notice that this limit imposes a precise and not trivial constraint on the minimum observable bandwidth of the idler photon: the behaviour described by Eq.(39) is indeed realized only for $\tau_{p} \ll \tau_{\mathrm{gvm}}$, or for $\Delta \Omega_{\mathrm{p}} \gg \Omega_{\mathrm{gvm}}$, so that it requires that the idler bandwidth

$$
\delta \Omega_{i} \simeq \frac{\eta}{1-\eta} \Delta \Omega_{\mathrm{p}} \gg \frac{\eta}{1-\eta} \Omega_{\mathrm{gvm}}=\Omega_{\mathrm{gvs}}
$$

\section{iii) Intermediate pump pulse:}

When $\tau_{\mathrm{gvm}} \ll \tau_{p} \ll \tau_{\mathrm{gvs}}^{\prime}$, the properties of the twin photons are actually intermediate between the two former cases, with the forward propagating signal photon replicating the pump spectrum, while the coherence properties of the backward propagating idler are determined by phase matching. These features are clearly exhibited by the central 
column (b) of Fig 4 , which plots a numerically computed example of the coherence functions for $\tau_{p}=0.04 \tau_{\mathrm{gvs}}^{\prime} \approx 4 \tau_{\mathrm{gvm}}$, short with respect to the transit time along the slab, but long enough that GVM does not play a relevant role.

The observed features are a straightforward consequence of the separable form $(29)$ of the biphoton amplitude which holds in this limit. Indeed, by using Eq. 29, , in the limit $\tau_{p} / \tau_{\mathrm{gvs}}^{\prime} \rightarrow 0, \tau_{\mathrm{gvm}} / \tau_{\mathrm{p}} \rightarrow 0$ we obtain:

$$
\begin{aligned}
G_{s}^{(1)}\left(\Omega, \Omega^{\prime}\right) & \rightarrow \frac{g^{2} \Omega_{\mathrm{gvs}}}{2} \tilde{\alpha}_{p}^{*}[\Omega(1-\eta)] \tilde{\alpha}_{p}\left[\Omega^{\prime}(1-\eta)\right] \\
G_{i}^{(1)}\left(\Omega, \Omega^{\prime}\right) & \rightarrow \frac{g^{2} \tau_{\mathrm{p}}}{\sqrt{2 \pi}} \operatorname{sinc}\left(\frac{\Omega}{\Omega_{\mathrm{gvs}}}\right) \operatorname{sinc}\left(\frac{\Omega^{\prime}}{\Omega_{\mathrm{gvs}}}\right) e^{i \frac{\Omega^{\prime}-\Omega}{\Omega_{\mathrm{gvs}}}}
\end{aligned}
$$

Thus in this case the signal spectrum is a replica of the broad pump spectrum $I_{s}(\Omega) \propto\left|\tilde{\alpha}_{p}[\Omega(1-\eta)]\right|^{2}$, while the idler spectrum is determined by the much narrower phase-matching function $I_{i}(\Omega) \propto \operatorname{sinc}^{2}\left(\frac{\Omega}{\Omega_{\mathrm{gvs}}}\right)$. Precisely, the signal spectrum is described by the same formula (38) as in the ultrashort pump case, while the idler spectral properties are described by the same formula (36) that holds in the CW pump limit. However, notice that in the present case the coherence properties are remarkably different, as the two coherence functions are perfectly symmetrical along the two diagonals $\Omega \pm \Omega^{\prime}$ : as can be easily inferred from Eqs. (41) and (42) the two coherence lengths are $l_{c o h, s} \approx \Delta \Omega_{\mathrm{p}}$ and $l_{c o h, i} \approx \Omega_{\mathrm{gvs}}$, i.e. they are equal to the respective spectral widths. This is in accordance with the separability of the biphoton state, which corresponds to single-mode, almost coherent reduced states for each of the two twin photon taken separately.

We conclude this section observing that the results $(36),(40)$ and $(42)$ implies that in any pumping regime the idler bandwidth cannot be narrower than the phase matching bandwidth $\Omega_{\mathrm{gvs}}$, a limitation that arises from the imperfect momentum conservation due to the finite length of the crystal slab.

\section{SCHMIDT NUMBER OF ENTANGLEMENT}

So far our considerations about the number of modes and the degree of entanglement of the system have been qualitative. A quantitative measure of the entanglement is offered by the so-called Schmidt number [19, 20, which is recognized to give an estimate of the number of Schmidt modes partecipating in the entangled state, i.e. of the effective dimensionality of the entanglement [21]. First of all, as usual, we consider the state conditioned to a photon count

$$
\left|\phi_{\mathrm{C}}\right\rangle=\int d \Omega_{s} d \Omega_{i} \psi\left(\Omega_{s}, \Omega_{i}\right) \hat{a}_{s}^{\dagger}\left(\Omega_{s}\right) \hat{a}_{i}^{\dagger}\left(\Omega_{i}\right)|0\rangle,
$$

where with respect to the true output state $(12)$, the vacuum term has been dropped. Then, we introduce the Schmidt number, as the inverse of the purity of the state of each separate subsystem

$$
\mathcal{K}=\frac{1}{\operatorname{Tr}\left\{\rho_{s}^{2}\right\}}=\frac{1}{\operatorname{Tr}\left\{\rho_{i}^{2}\right\}}
$$

where $\rho_{s}, \rho_{i}$ are the reduced density matrix of the signal and idler, e.g. $\rho_{s}=\operatorname{T} r_{i}\left\{\left|\phi_{\mathrm{C}}\right\rangle\left\langle\phi_{\mathrm{C}}\right|\right\}$. For a two-particle state of the form (43), the Schmidt number can be calculated via an integral formula, as e.g derived in [4] (see also [5]),

$$
\mathcal{K}=\frac{\mathcal{N}^{2}}{B}
$$

where

$$
\begin{aligned}
\mathcal{N} & =\int d \Omega G_{s}^{(1)}(\Omega, \Omega)=\int d \Omega G_{i}^{(1)}(\Omega, \Omega) \\
B & =\int d \Omega \int d \Omega^{\prime}\left|G_{s}^{(1)}\left(\Omega, \Omega^{\prime}\right)\right|^{2}=\int d \Omega \int d \Omega^{\prime}\left|G_{i}^{(1)}\left(\Omega, \Omega^{\prime}\right)\right|^{2}
\end{aligned}
$$

As can be easily checked, $\mathcal{N}$ is the espectation value (first order moment) of the photon number operator $\hat{N}_{j}=$ $\int d \Omega \hat{I}_{j}(\Omega)$ in either the signal or idler arm

$$
\mathcal{N}=\left\langle\hat{N}_{s}\right\rangle=\left\langle\hat{N}_{i}\right\rangle
$$


The quantity at denominator is instead linked to the second order moment of the photon number. By performing the integral of Eq. 33 over the two spectral arguments, one gets:

$$
B=\left\langle: \hat{N}_{j}^{2}:\right\rangle-\left\langle\hat{N}_{j}\right\rangle^{2} \quad(j=s, i)
$$

where the symbol : : indicates normal ordering. In terms of the normalized $g^{(2)}$ coefficient:

$$
g^{(2)}=\frac{\left\langle: \hat{N}_{j}^{2}:\right\rangle}{\left\langle\hat{N}_{j}\right\rangle^{2}}=1+\frac{1}{\mathcal{K}}
$$

In this way, as recognized in [22, 23, the Schmidt number can be related to measurable statistical properties of light. In particular, formula $(50)$ is well know to describe the statistics of multi-mode thermal light, with $\mathcal{K}$ playing the role of the "degeneracy factor" characterizing the effective number of independent modes in a thermal beam.

Figure 5 shows our results for the Schmidt number. The solid lines plot the "exact" results, where $\mathcal{K}$ has been calculated by numerically performing the integrals involved in 446), 477, with the phase matching calculated via the complete Sellmeier relations. The red dashed lines in plot (a) and (b) are asymptotic behaviours, analytically derived by exploiting the linear approximation for phase matching. In particular, by using the approximated formula (35) for the coherence function, and performing the integrals involved in (46) and (47), one obtains the limit of the Schmidt number for a long pump pulse

$$
\mathcal{K} \stackrel{\tau_{p} \gg \tau_{\text {gvs }}^{\prime}}{\longrightarrow} \frac{3}{2} \sqrt{\frac{\pi}{2}} \frac{\Omega_{\text {gvs }}^{\prime}}{\Delta \Omega_{p}}
$$

For an ultrashort pump pulse, the asymptotic behaviour of $\mathcal{K}$ is calculated by using formula (39) or (37), for either the signal or the idler coherence function (identical results are indeed obtained). In this case

$$
\mathcal{K} \stackrel{\tau_{p} \ll \tau_{\text {gvm }}}{\longrightarrow} \frac{1}{1-\eta} \sqrt{\frac{2}{\pi}} \frac{\Delta \Omega_{p}}{\Omega_{\mathrm{gvm}}}
$$

The calculated asymptotes are well in accordance with our qualitative estimates of the number of modes in Sec III. based on the ratio between the spectral bandwidth and the coherence length.

This shape of the curve, showing a minimum of $\mathcal{K}$ for a given value of the pump bandwidth and linear asymptotes at small and large values of the bandwidth, is commonplace, with a qualitatively similar curve characterizing also the co-propagating case in either temporal [5] or spatial [24] or even spatio-temporal [4] domains. The novelty here is that the minimum value of $\mathcal{K}$ is very close to unity, and remains close to unity for a rather large range of $\Delta \Omega_{p}$ (see panel (c) in Fig 5). This represents indeed a big difference compared to the copropagating case, where in order to generate separable biphotons very special matching conditions have to be chosen, corresponding to a zero group velocity mismatch between the pump and one of the twin photons, which can be realized only in type II interactions [25, 26].

In the backward propagating case the conditions for separability are very easily approached, and rely entirely on the fact that $\eta=\tau_{\mathrm{gvm}} / \tau_{\mathrm{gvs}}^{\prime}$ is naturally a very small quantity, because the temporal separations $\tau_{\mathrm{gvm}}, \tau_{\mathrm{gvs}}^{\prime}$ between the co-propagating and the counterpropagating waves are on well separated time scales.

Indeed, a more refined calculation shows that the minimum value of $\mathcal{K}$, reached for a pump duration intermediate between $\tau_{\mathrm{gvm}}$ and $\tau_{\mathrm{gvs}}^{\prime}$ is $K_{\min }=1+O(\eta)$. This is also confirmed by analytical calculations of the Schmidt number, reported in detail elsewhere 27], performed by means of a Gaussian approximation of the sinc function of phase matching, similarly to what done in [25]. These calculations (plotted as the blu dash-dot line in Fig 5 ;) show that the minimum of $\mathcal{K}$ is

$$
\mathcal{K}_{\min }=\frac{1+\eta}{1-\eta} \approx 1+2 \eta
$$

reached for $\Delta \Omega_{p}=\sqrt{3 \Omega_{\mathrm{gvs}}^{\prime} \Omega_{\mathrm{gvm}}}$ This result suggest that a higher degree of purity of the reduced states can be achieved as the GVM between the two forward propagating is reduced. This is confirmed by the examples in Fig 6 , which compares different crystals and phase matching conditions. Notice that a small GVM corresponds to a higher degree of purity, as in the copropagating case, but that in the present case the condition for separability is much less demanding, as it does not require a vanishing GVM, but just that that $\tau_{\mathrm{gvm}}$ is small compared to sum of the inverse of group velocities $\tau_{\mathrm{gvs}}^{\prime}$, which is always verified to some extent. 


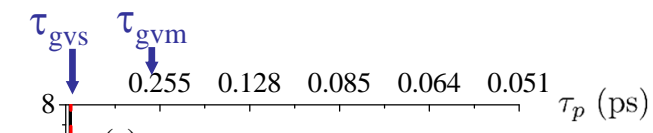

(a)
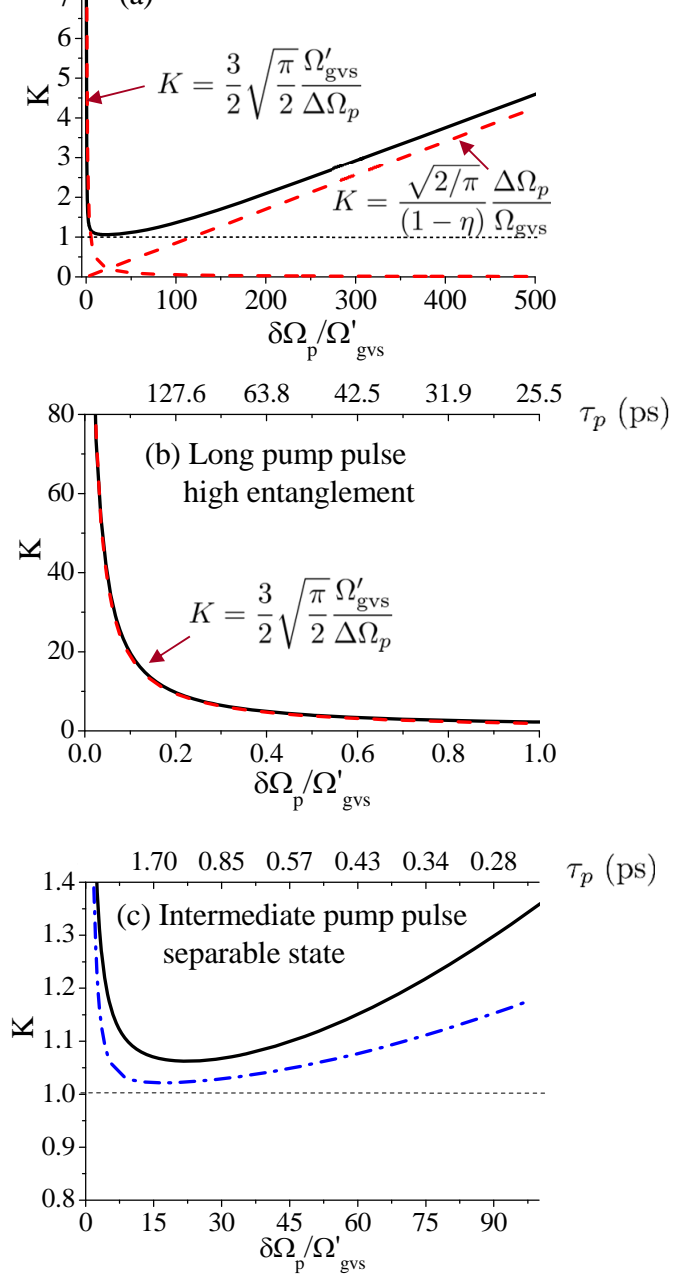

FIG. 5: Schmidt number, as a function of the pump spectral bandwidth (lower axis) or duration (upper axis). (b) and (c) are insets of (a), showing the transition from high entanglement for a long pump $\tau_{p} \gg \tau_{\text {gvs }}^{\prime}$ to an almost separable state for $\tau_{\mathrm{gvs}}^{\prime} \gg \tau_{p} \gg \tau_{\mathrm{gvm}}$. The red dashed lines in (a) and (b) are the calculated asymptotic behaviours, the blu dash-dot line in (c) is the result of a Gaussian approximation. $4 \mathrm{~mm}$ PPKTP A in Fig 2 , with $\tau_{\mathrm{gvs}}^{\prime}=25.5 \mathrm{ps} \tau_{\mathrm{gvm}}=0.27 \mathrm{ps}, \eta=0.01$, other parameters as in Fig 3

\section{INTERPRETATION: THE BIPHOTON CORRELATION IN THE TEMPORAL DOMAIN}

An alternative insight into the issue of separability vs entanglement is provided by the analysis of the biphoton correlation in the temporal domain. We consider

$$
\phi\left(t_{s}, t_{i}\right)=\left\langle\hat{A}_{s}^{\text {out }}\left(t_{s}\right) \hat{A}_{i}^{\text {out }}\left(t_{i}\right)\right\rangle=\int \frac{d \Omega_{s}}{\sqrt{2 \pi}} \int \frac{d \Omega_{i}}{\sqrt{2 \pi}} e^{-i\left(\Omega_{s} t_{s}+\Omega_{i} t_{i}\right)} e^{i k_{s}\left(\Omega_{s}\right) l_{c}} \psi\left(\Omega_{s}, \Omega_{i}\right),
$$

which is proportional to the probability amplitude of finding a signal and an idler photons at their crystal end faces at times $t_{s}, t_{i}$. By using the linear approximation for phase matching (15) and performing the simple Fourier transformations involved in (54) we obtain:

$$
\phi\left(\bar{t}_{s}, \bar{t}_{i}\right)=\frac{g e^{i k_{s} l_{c}}}{2 \tau_{\mathrm{gvs}}} \alpha_{p}\left(\bar{t}_{s}+\eta \frac{\bar{t}_{s}-\bar{t}_{i}}{1-\eta}\right) \operatorname{Rect}\left(\frac{\bar{t}_{s}-\bar{t}_{i}}{2 \tau_{\mathrm{gvs}}}\right)
$$




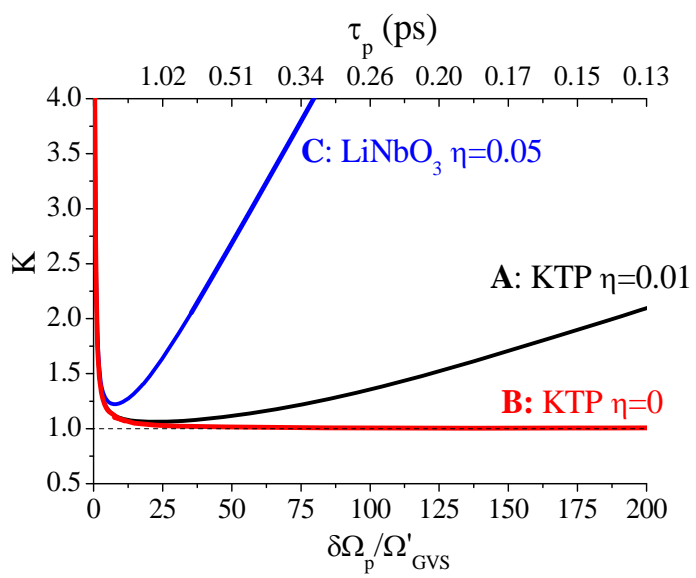

FIG. 6: Role of GVM in determining the purity of the state: Schmidt number for different crystals and/or different phase matching conditions, corresponding to the points $\mathbf{A}, \mathbf{B}, \mathbf{C}$ in Fig 2 A) $4 \mathrm{~mm} \mathrm{KTP}$ with $\tau_{\mathrm{gvm}}=0.27 \mathrm{ps}, \tau_{\mathrm{gvs}}^{\prime}=25.5 \mathrm{ps}, \rightarrow \eta=0.01$

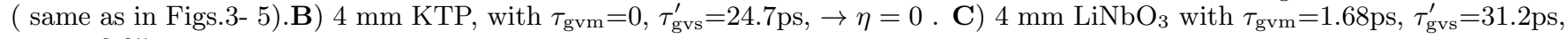
$\rightarrow \eta=0.05$

where

$$
\operatorname{Rect}(x)=\left\{\begin{array}{lc}
1 & \text { for } \\
0 & \text { elsewhere }
\end{array} \quad x\left(-\frac{1}{2}, \frac{1}{2}\right),\right.
$$

is the box function of unitary width. The barred arguments $\bar{t}_{s}, \bar{t}_{i}$ denote time intervals measured starting from the arrival times of the centers of the signal/idler wavepackets. Precisely, $\bar{t}_{s, i}=t_{s, i}-t_{A s, i}$, where

$$
\begin{aligned}
& t_{A s}=\left(k_{s}^{\prime}+k_{p}^{\prime}\right) \frac{l_{c}}{2}=t_{A p}-\left(k_{p}^{\prime}-k_{s}^{\prime}\right) \frac{l_{c}}{2} \\
& t_{A i}=\left(k_{i}^{\prime}+k_{p}^{\prime}\right) \frac{l_{c}}{2}=t_{A p}-\left(k_{p}^{\prime}-k_{i}^{\prime}\right) \frac{l_{c}}{2}
\end{aligned}
$$

where $t_{A p}=k_{p}^{\prime} l_{c}$ is the time when the center of the pump pulse exits the crystal slab. Figure 7 shows three examples of the temporal correlation function (55).

The general formula (55) can be simplified in the limit where the pump is long with respect to $\tau_{\text {gvm }}$, i.e. in the quasi CW or intermediate limits 21, 28, where it takes the form

$$
\phi\left(\bar{t}_{s}, \bar{t}_{i}\right) \stackrel{\tau_{p} \gg \tau_{G V M}}{\simeq} g e^{i k_{s} l_{c}} \alpha_{p}\left(\bar{t}_{s}\right) \frac{1}{2 \tau_{\mathrm{gvs}}} \operatorname{Rect}\left(\frac{\bar{t}_{i}-\bar{t}_{s}}{2 \tau_{\mathrm{gvs}}}\right)
$$

Indeed, when the the pump pulse is long with respect to $\tau_{\mathrm{gvm}}$, we have $\alpha_{p}\left(\bar{t}_{s}+\eta \frac{\bar{t}_{s}-\bar{t}_{i}}{1-\eta}\right) \approx \alpha_{p}\left(\bar{t}_{s}\right)$, because $\left|\bar{t}_{s}-\bar{t}_{i}\right|$ is limited by the box function to values smaller than $\tau_{\mathrm{gvs}}$, so that $\eta \frac{\left|\bar{t}_{s}-\bar{t}_{i}\right|}{1-\eta}=\frac{\tau_{\mathrm{gvm}}}{\tau_{\mathrm{gvs}}}\left|\bar{t}_{s}-\bar{t}_{i}\right| \leq \tau_{\mathrm{gvm}} \ll \tau_{p}$.

Formula $(60)$ shows that in the limit of a negligible $G V M$, the distribution of separations $\bar{t}_{s}-\bar{t}_{i}$ between the arrival times of the twin photons is entirely described by the box function of width $2 \tau_{\mathrm{gvs}}$. This form of the temporal correlation clearly reflects the spontaneous character of the process, where photon pairs can be generated at any point of the crystal with uniform probability. Thus, assuming for simplicity that the the twins travel with the same group velocities $v_{g s}=v_{g i}$, the separation between their arrival times ranges with uniform probability from zero, when the two photons are generated at the center of the crystal up to $\pm \tau_{\mathrm{gvs}}=l_{c} / v_{g}$, when they are generated at each of the end faces. ${ }^{1}$

\footnotetext{
${ }^{1}$ Precisely, when the two photons are generated at the crystal center $t_{s}-t_{i}=t_{A s}-t_{A i}=\left(k_{s}^{\prime}-k_{i}^{\prime}\right) l_{c} / 2 \approx 0$, and the delay between their arrival times ranges uniformly between i) $t_{s}-t_{i}=t_{A s}-t_{A i}-\tau_{\mathrm{gvs}}=-k_{i}^{\prime} l_{c}$, when they are generated at the right end face of the slab, and ii) $t_{s}-t_{i}=t_{A s}-t_{A i}+\tau_{\mathrm{gvs}}=k_{s}^{\prime} l_{c}$ when the photon pair is generated at the left end face.
} 


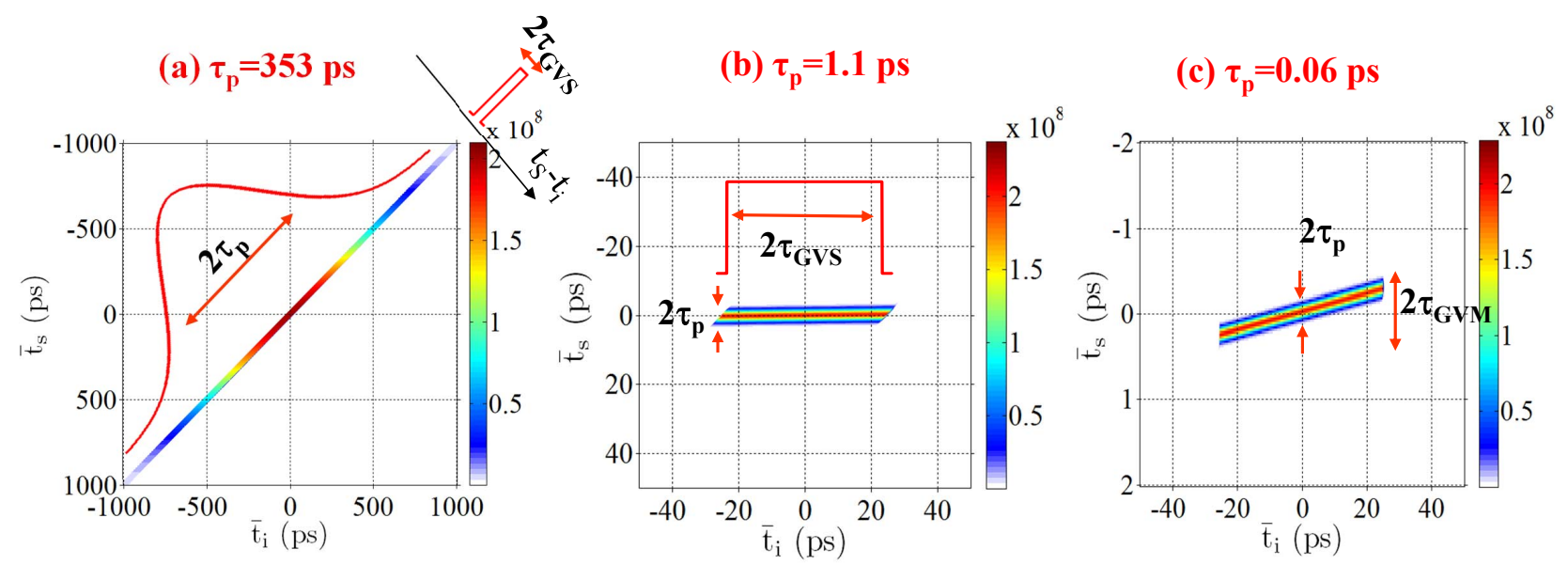

FIG. 7: (Color online) Temporal correlation of twin photons $\left|\phi\left(\bar{t}_{i}, \bar{t}_{s}\right)\right|$, given by Eq. 55 , plotted in the plane $\left(\bar{t}_{i}, \bar{t}_{s}\right)$. (a)High entanglement case, with $\mathcal{K} \simeq 26$, for a quasi CW pump $\tau_{\mathrm{p}}=14 \tau_{\text {gvs }}^{\prime}$. (b)Almost separable case with $\mathcal{K} \simeq 1.06$, for an intermediate pump $\tau_{\mathrm{p}}=0.04 \tau_{\mathrm{gvs}}^{\prime}=4 \tau_{\mathrm{gvm}}$. (c) Ultrashort pulse $\tau_{\mathrm{p}}=0.22 \tau_{\mathrm{gvm}}$, corresponding to an entangled state with $\mathcal{K} \simeq 4$. Same KTP crystal as in Fig 5

The CW pump limit (Fig $7 \mathrm{7}$ ) corresponds to the situation where the pump pulse is much longer than the maximal temporal separation $\tau_{\mathrm{gvs}}$ between the twins. In this case, the usual picture of the temporal entanglement of twin photons holds: the time when a signal or idler photon is individually detected has a large undeterminacy, because a photon pair can be generated at any time along the pump pulse. However, from the arrival time of one of the members of the pair one can infer the arrival time of the other with a much smaller uncertainty $\tau_{\text {gvs }}$, which represents the mean uncertainty in the arrival time of one photon provided its twin have been detected, i.e. the correlation time. This kind of correlation is basically what predicted in Ref. [13] for a strictly monochromatic pump.

However, when the pump pulse shorten below $\tau_{\text {gvs }}$ (Fig.7b) this description ceases to be valid, because the localization of the pump pulse provides a timing information on the arrival time of the signal that is more precise than the uncertainty in the temporal separation of the twins. Indeed when the pump pulse is much shorter than $\tau_{\mathrm{gvs}}$, but still long enough that GVM is negligible, the signal wavepacket overlaps almost exactly with the pump pulse during propagation, and the uncertainty in the arrival time of the signal is just the pulse duration. This is much smaller than the conditional uncentainty $\tau_{\mathrm{gvs}}$ by which the arrival time of the idler can be inferred from that of the signal, so that the arrival times of the members of the pair appear completely uncorrelated. Indeed, the temporal correlation in Fig.7b is approximately:

$$
\phi\left(\bar{t}_{s}, \bar{t}_{i}\right) \simeq g e^{i k_{s} l_{c}} \alpha_{p}\left(\bar{t}_{s}\right) \frac{1}{2 \tau_{\mathrm{gvs}}} \operatorname{Rect}\left(\frac{\bar{t}_{i}}{2 \tau_{\mathrm{gvs}}}\right),
$$

which is a factorable function of $\bar{t}_{s}, \bar{t}_{i}$.

Notice that when the pump pulse is so short that GVM starts to be important (Fig 77), there is again a loss of absolute timing information, because in this case the arrival time of the signal cannot be inferred from that of the pump with a precision better than $\tau_{\mathrm{gvm}}$. In contrast, the arrival time of the signal conditioned to a photon count in the idler arm can be predicted within the short pump duration $\tau_{p}$, and the state becomes again entangled. This can be better understood by looking the correlation function (55), which for $\tau_{p} \ll \tau_{\text {gvm }}$ can be rewritten as

$$
\begin{aligned}
\phi\left(\bar{t}_{s}, \bar{t}_{i}\right) & =\frac{g e^{i k_{s} l_{c}}}{2 \tau_{\mathrm{gvs}}} \alpha_{p}\left(\frac{\bar{t}_{s}-\eta \bar{t}_{i}}{1-\eta}\right) \operatorname{Rect}\left(\frac{\bar{t}_{s}-\bar{t}_{i}}{2 \tau_{\mathrm{gvs}}}\right) \\
& \simeq \frac{g e^{i k_{s} l_{c}}}{2 \tau_{\mathrm{gvs}}} \alpha_{p}\left(\frac{\bar{t}_{s}-\eta \bar{t}_{i}}{1-\eta}\right) \operatorname{Rect}\left(\frac{\bar{t}_{s}}{2 \tau_{\mathrm{gvm}}}\right) .
\end{aligned}
$$

where the last line has been obtained by substituting $\bar{t}_{i}=\bar{t}_{s} / \eta$ inside the argument of the box function (valid because the pump profile is much narrower than both $\tau_{\mathrm{gvs}}$ and $\tau_{\mathrm{gvm}}$ ). From formula 62 we see that, provided that an idler photon is detected, say at time $\bar{t}_{i}$, the arrival time of the signal can be predicted as $\bar{t}_{s}=\eta \bar{t}_{i}$ within the narrow uncertainty of the pump duration $\tau_{p}$ (see also Fig.7p). However when the idler is not detected, the overall uncertainty in the signal arrival time is the larger width $\tau_{\mathrm{gvm}}$ of the box function. Clearly this argument predicts an entangled state, with the number of modes scaling as $\tau_{\mathrm{gvm}} / \tau_{p}$, in agreement with formula 52 . 


\section{CONCLUSIONS}

In this work we provided a detailed theoretical analysis of the effect of the pump spectral properties on the quantum correlation of counterpropagating photons generated by SPDC in a periodically poled crystal.

In particular, for increasing spectral bandwidths of the pump (descreasing pump durations), we demontrated a remarkable transition from a high-dimensional entangled state, to an almost separable state. The transition occurs when the pulse duration shorten below the characteristic transit time $\tau_{\mathrm{gvs}}=\frac{l_{c}}{2 v_{g s}}+\frac{l_{c}}{2 v_{g i}} \approx \frac{l_{c}}{2 v_{g p}}+\frac{l_{c}}{2 v_{g i}}$ of light along the crystal slab. This long temporal scale is a unique characteristic of the counterpropagating geometry, being associated to the delay between the times at which the counterpropagating photons, generated at some point along the slab, appear at their exit faces. The temporal correlation (temporal entanglement) is again restored for pump durations below the short temporal delay occurring between the co-propagating waves because of their different group velocities. The natural existence of such separated time scales ensures the possibility of generating high purity single photon (i.e. a separable two-photon state), under very general conditions, which differs drastically from the usual co-propagating geometry [26].

These conclusions have been supported throught the paper by the analysis of the Schmidt number in Sec IV], and by analytical and numerical evaluations of the spectral and temporal correlation function (Sec.II. SecV).

The study of the maginal statistics of photons in Sec. III has revealed several non-trivial features:

While for a long pump pulse twin photons have the same spectrum and the same coherence properties, in the regime of separability they exhibit very different features. In particular, the properties of the counterpropagating idler are entirely determined by the phase matching in the medium, so that we can say that they reflect the momentum conservation in the process. On the other side, the spectro-temporal properties of the signal are a replica of those of the co-propagating pump laser pulse, and rather reflect the energy conservation.

For an ultrashort pump pulse, our quantum analysis has retrieved results analogue to what predicted in the classical description of the MOPO [8, 9], but with some additional limitation. At difference with the MOPO prediction [8, our results impose a precise inferior limit to the observable bandwidth of the backward idler photon, which cannot be narrower than the phase matching bandwidth $\Omega_{\mathrm{gvs}}$. Clearly, our analysis is limited to SPDC, but we notice that, to our knowledge, measurements of the spectrum of the backward wave in the MOPO have been limited by the spectrometer resolution [10], so that our findings may open a question about the effective bandwidth of the backward wave.

\section{Appendix A: Approximations for the biphoton amplitude}

In this Appendix we derive the approximated forms of the biphoton amplitude used in the text, which holds in the various pump regimes. In all the cases we make use of the linear approximation for phase matching (15), based on the assumption that the bandwidths in play are narrow so that dispersion can be neglected. Under this approximation, the general espression 10 of the biphoton amplitude becomes

$$
\psi\left(\Omega_{s}, \Omega_{i}\right)=\frac{g}{\sqrt{2 \pi}} \tilde{\alpha}_{p}\left(\Omega_{s}+\Omega_{i}\right) V\left(\frac{\Omega_{s}}{\Omega_{\mathrm{gvm}}}+\frac{\Omega_{i}}{\Omega_{\mathrm{gvs}}^{\prime}}\right)
$$

where for brevity of notation we introduced the phase matching function $V(s)=\operatorname{sinc}(s) e^{i s}$.

We consider first the limit of a CW pump (21). Since the pump bandwith is much narrower than the bandwidths $\Omega_{\mathrm{gvs}}^{\prime}$ and $\Omega_{\mathrm{gvm}}$ of phase matching, the presence of the pump Fourier amplitude term forces $\Omega_{s}=-\Omega_{i}$ into the phase matching function. As a result

$$
\begin{aligned}
\lim _{\tau_{p} / \tau_{\mathrm{gvs}}^{\prime} \rightarrow \infty} \psi\left(\Omega_{s}, \Omega_{i}\right) & =\frac{g}{\sqrt{2 \pi}} \tilde{\alpha}_{p}\left(\Omega_{s}+\Omega_{i}\right) V\left(-\frac{\Omega_{s}}{\Omega_{\mathrm{gvs}}}\right) \\
& =\frac{g}{\sqrt{2 \pi}} \tilde{\alpha}_{p}\left(\Omega_{s}+\Omega_{i}\right) V\left(\frac{\Omega_{i}}{\Omega_{\mathrm{gvs}}}\right)
\end{aligned}
$$

where we used the relation $1 / \Omega_{\mathrm{gvs}}=1 / \Omega_{\mathrm{gvs}}^{\prime}-1 / \Omega_{\mathrm{gvm}}$, according to the definitions $16,-18$.

The limit 24) of an ultrashort pump is also straightforward. In this case the bandwidths of phase matching are assumed to be much narrower than the pump bandwidth $\Omega_{\mathrm{gvs}}^{\prime} \ll \Omega_{\mathrm{gvm}} \ll \Delta \Omega_{\mathrm{p}}$, so that the phase matching function has a narrow peak, which on the slow scale of variation of the pump forces $\Omega_{i}=-\eta \Omega_{s}$, or $\Omega_{s}=-\Omega_{i} / \eta$ inside the 
pump argument. Therefore

$$
\begin{aligned}
\lim _{\frac{\tau_{p}}{\tau_{\mathrm{gvm}} \rightarrow 0}} \psi\left(\Omega_{s}, \Omega_{i}\right) & =\frac{g}{\sqrt{2 \pi}} \tilde{\alpha}_{p}\left[\Omega_{s}(1-\eta)\right] V\left(\frac{\Omega_{s}}{\Omega_{\mathrm{gvm}}}+\frac{\Omega_{i}}{\Omega_{\mathrm{gvs}}^{\prime}}\right) \\
& =\frac{g}{\sqrt{2 \pi}} \tilde{\alpha}_{p}\left[-\Omega_{i} \frac{1-\eta}{\eta}\right] V\left(\frac{\Omega_{s}}{\Omega_{\mathrm{gvm}}}+\frac{\Omega_{i}}{\Omega_{\mathrm{gvs}}^{\prime}}\right)
\end{aligned}
$$

The intermediate pump limit $(28)$ where $\Omega_{\text {gvs }}^{\prime} \ll \Delta \Omega_{\mathrm{p}} \ll \Omega_{\text {gvm }}$ is a bit more involved. We remind that the existence of this limit also requires $\eta=\Omega_{\text {gvs }}^{\prime} / \Omega_{\text {gvm }} \ll 1$, which is in practice always verified to some extent. By introducing the pump frequency $\Omega_{p}=\Omega_{s}+\Omega_{i}$, we recast the argument of the sinc function

$$
\frac{\Omega_{s}}{\Omega_{\mathrm{gvm}}}+\frac{\Omega_{i}}{\Omega_{\mathrm{gvs}}^{\prime}}=\frac{\Omega_{p}}{\Omega_{\mathrm{gvm}}}+\Omega_{i}\left(\frac{1}{\Omega_{\mathrm{gvs}}^{\prime}}-\frac{1}{\Omega_{\mathrm{gvm}}}\right) \approx \frac{\Omega_{i}}{\Omega_{\mathrm{gvs}}}
$$

where the term $\Omega_{p} / \Omega_{\text {gvm }}$ has been neglected because is on the order $\Delta \Omega_{\mathrm{p}} / \Omega_{\mathrm{gvm}} \ll 1$ Concerning the pump amplitude we recast it as:

$$
\begin{aligned}
\tilde{\alpha}_{p}\left(\Omega_{s}+\Omega_{i}\right) & =\tilde{\alpha}_{p}\left[\Omega_{s}(1-\eta)+\left(\frac{\Omega_{s}}{\Omega_{\mathrm{gvm}}}+\frac{\Omega_{i}}{\Omega_{\mathrm{gvs}}^{\prime}}\right) \Omega_{\mathrm{gvs}}^{\prime}\right] \\
& \approx \tilde{\alpha}_{p}\left[\Omega_{s}(1-\eta)\right]
\end{aligned}
$$

where the approximation in the second second line holds because $\left(\frac{\Omega_{s}}{\Omega_{\mathrm{gvm}}}+\frac{\Omega_{i}}{\Omega_{\mathrm{gvs}}^{\prime}}\right)$ is the argument of the sinc function (see Eq. A1), so that it is limited to values inside the bandwidth of the sinc, say on the order $\simeq 10$. Provided thar $\Omega_{\text {gvs }}^{\prime} / \Delta \Omega_{\mathrm{p}}$ is small enough, this term becomes therefore negligible. With this in mind we can write the limiting behaviour of the biphoton amplitude:

$$
\begin{aligned}
\lim _{\substack{\tau_{p} / \tau_{\mathrm{gvs}}^{\prime} \rightarrow 0 \\
\tau_{\mathrm{gvm}} / \tau_{\mathrm{p}} \rightarrow 0}} \psi\left(\Omega_{s}, \Omega_{i}\right) & =\frac{g}{\sqrt{2 \pi}} \tilde{\alpha}_{p}\left[\Omega_{s}(1-\eta)\right] e^{i \frac{\Omega_{s}}{\Omega_{\mathrm{gvm}}}} \times \operatorname{sinc}\left(\frac{\Omega_{i}}{\Omega_{\mathrm{gvs}}}\right) e^{i \frac{\Omega_{i}}{\Omega_{\mathrm{gvs}}}} \\
& \approx \frac{g}{\sqrt{2 \pi}} \tilde{\alpha}_{p}\left[\left(\Omega_{s}\right) e^{i \frac{\Omega_{s}}{\Omega_{\mathrm{gvm}}}} \times \operatorname{sinc}\left(\frac{\Omega_{i}}{\Omega_{\mathrm{gvs}}}\right) e^{i \frac{\Omega_{i}}{\Omega_{\mathrm{gvs}}}} .\right.
\end{aligned}
$$

where the approximation in the last line is not mandatory, but could be useful in order to get consistent results, because clearly this limit can be realized only for $\eta=\tau_{\mathrm{gvm}} / \tau_{\mathrm{gvs}}^{\prime} \rightarrow 0$.

[1] C. K. Hong, Z. Y. Ou, and L. Mandel, Measurement of Subpicosecond Time Intervals between Two Photons by Interference, Phys. Rev. Lett. 59, 2044 (1987).

[2] A. Gatti, E. Brambilla, L. Caspani, O. Jedrkiewicz and L. A. Lugiato, Phys.Rev. Lett. 102, 223601 (2009).

[3] O. Jedrkiewicz, J.-L. Blanchet, E. Brambilla, P. Di Trapani and A. Gatti, Phys. Rev. Lett. 108 , 253904 (2012)

[4] A. Gatti, E. Brambilla, T. Corti and D. M. Horoshko, Dimensionality of the spatio-temporal entanglement of PDC photon pairs, Phys. Rev. A 86, 053803 (2012).

[5] Y. M. Mikhailova,P. A. Volkov and M. V. Fedorov,Phys. Rev. A 78, 062327 (2008) .

[6] Malte Avenhaus, Maria V. Chekhova, Leonid A. Krivitsky, Gerd Leuchs and Christine Silberhorn, Experimental verification of high spectral entanglement for pulsed waveguided spontaneous parametric down-conversion, Phys. Rev. A 79,043836 (2009)

[7] S. E. Harris, Proposed backward wave oscillation in the infrared,Appl. Phys. Lett. 9, 114116 (1966).

[8] Carlota Canalias and Valdas Pasiskevicius, Mirrorless optical parametric oscillators, Nature photonics 1, 459 (2007).

[9] Gustav Strmqvist, Valdas Pasiskevicius, Carlota Canalias, Pierre Aschieri, Antonio Picozzi, and Carlos Montes, Temporal coherence in mirrorless optical parametric oscillators, J. Opt. Soc. Am. B 29, 1194 ( 2012).

[10] Valdas Pasiskevicius, Gustav Strmqvist, Fredrik Laurell, Carlota Canalias, Quasi-phase matched nonlinear media: Progress towards nonlinear optical engineering, Optical Materials 34513523 (2012).

[11] Yujie J. Ding and Jacob B. Khurgin, Backward Optical Parametric Oscillators and Amplifiers, IEEE J. of Quantum Electronics 32,1574 (1996).

[12] Tommaso Corti, Enrico Brambilla and Alessandra Gatti, Critical behaviour of the temporal correlation and coherence of counterpropagating twin beams, preprint.

[13] Toshiaki Suhara and Makoto Ohno, Quantum Theory Analysis of Counterpropagating Twin Photon Generation by Parametric Downconversion, IEEE J. OF QUANTUM ELECTRONICS 46, 1739 (2010) 
[14] A. Christ, A. Eckstein, P. J. Mosley and C. Silberhorn,Pure single photon generation by type-IPDC with backward-wave amplification, Opt. Expr. 17, 3441 (2009)

[15] M. C. Booth, M. Atature, G. Di Giuseppe, A. V. Sergienko, B. E. A. Saleh, and M. C. Teich, Counter-propagating entangled photons from a waveguide with periodic nonlinearity, Phys. Rev. A 66, 02381502323 (2002).

[16] E. Brambilla, O. Jedrkiewicz, P. Di Trapani, A. Gatti, Space-time coupling in upconversion of broadband down-converted light, J. Opt. Soc. Am. B 31, 1383 (2014).

[17] Robert W. Boyd, Nonlinear Optics, 3rd Edition, Chapter 2, p.84, Elsevier ( 2008)

[18] David Nikogosyan, Nonlinear Optical Crystals: A Complete Survey, Springer New York (2005).

[19] A. Ekert,P.L. Knight, American Journal of Physics, 63, 415-423 (1995)

[20] S. Parker, S. Bose, and M. B. Plenio, Phys. Rev. A, 61, 032305 (2000).

[21] M.P Van Exter, A. Aiello, S.S.R. Oemrawsingh, G. Nienhuis and J.P. Woerdman, Phys. Rev. A 74012309 (2006).

[22] K. Laiho, A. Christ, K. N. Cassemiro, and C. Silberhorn, Testing spectral filters as Gaussian quantum optical channels, Opt. Lett 36, 1476 (2011).

[23] Andreas Christ, Kaisa Laiho, Andreas Eckstein, Katiscia N Cassemiro and Christine Silberhorn, Probing multimode squeezing with correlation functions, New J. of Phys. 13, 033027 (2011).

[24] C. K. Law and J. H. Eberly, Analysis and Interpretation of High Transverse Entanglement in Optical Parametric Down Conversion, Phys. Rev. Lett. 92, 127903 (2004) .

[25] Grice W P, U'Ren A B and Walmsley I A Phys. Rev. A 64063815 (2001)

[26] Mosley P J, Lundeen J S, Smith B J, Wasylczyk P, U'Ren A B, Silberhorn C and Walmsley I A, Heralded Generation of Ultrafast Single Photons in Pure Quantum States, Phys. Rev. Lett. 100133601 (2008 ); P. J. Mosley, J. S. Lundeen, B. J. Smith, and I. A. Walmsley, Conditional preparation of single photons using parametric downconversion: a recipe for purity, New J. Phys. 10, 093011 (2008).

[27] E. Brambilla, A.Gatti, The Schmidt number of counterpropagatin twin photons, in preparation 